\title{
Aggressive Surgical Effort and Improved Survival in Advanced-Stage Ovarian Cancer
}

\author{
Giovanni D. Aletti, MD, Sean C. Dowdy, MD, Bobbie S. Gostout, MD, Monica B. Jones, MD, \\ C. Robert Stanhope, MD, Timothy O. Wilson, MD, Karl C. Podratz, MD, PhD, and \\ William A. Cliby, MD
}

OBJECTIVE: Residual disease after initial surgery for ovarian cancer is the strongest prognostic factor for survival. However, the extent of surgical resection required to achieve optimal cytoreduction is controversial. Our goal was to estimate the effect of aggressive surgical resection on ovarian cancer patient survival.

METHODS: A retrospective cohort study of consecutive patients with International Federation of Gynecology and Obstetrics stage IIIC ovarian cancer undergoing primary surgery was conducted between January 1, 1994, and December 31, 1998. The main outcome measures were residual disease after cytoreduction, frequency of radical surgical resection, and 5-year disease-specific survival.

RESULTS: The study comprised 194 patients, including 144 with carcinomatosis. The mean patient age and follow-up time were 64.4 and 3.5 years, respectively. After surgery, 131 $(67.5 \%)$ of the 194 patients had less than $1 \mathrm{~cm}$ of residual disease (definition of optimal cytoreduction). Considering all patients, residual disease was the only independent predictor of survival; the need to perform radical procedures to achieve optimal cytoreduction was not associated with a decrease in survival. For the subgroup of patients with carcinomatosis, residual disease and the performance of radical surgical procedures were the only independent predictors. Disease-specific survival was markedly improved for patients with carcinomatosis operated on by surgeons who most frequently used radical procedures compared with those least likely to use radical procedures (44\% versus $17 \%, P<.001$ ).

CONCLUSION: Overall, residual disease was the only independent predictor of survival. Minimizing residual

From the Department of Obstetrics and Gynecology, Mayo Clinic, Rochester, Minnesota.

Corresponding author: William A. Cliby, MD, Department of Obstetrics and Gynecology, Mayo Clinic, 200 First Street SW, Rochester, MN 55905.

(C) 2005 by The American College of Obstetricians and Gynecologists. Published by Lippincott Williams \& Wilkins.

ISSN: 0029-7844/05 disease through aggressive surgical resection was beneficial, especially in patients with carcinomatosis.

(Obstet Gynecol 2006;107:77-85)

\section{LEVEL OF EVIDENCE: II-2}

$\mathrm{n}$ the United States, ovarian cancer is the leading cause of death from gynecologic malignancy, with 16,210 deaths anticipated to occur in $2005 .{ }^{1}$ The majority of women present with advanced intraabdominal disease and subsequently low cure rates. Since Griffiths' ${ }^{2}$ publication 30 years ago, the extent of cytoreductive surgery and the amount of residual disease after primary surgery have been considered the most important factors influencing the survival of patients with advanced ovarian cancer. The premise that low residual disease after primary surgery results in improved survival rates has held true in nearly every large retrospective and prospective publication to date. ${ }^{3,4}$ Accordingly, the concept of optimal cytoreduction has evolved over time and now generally applies to residual lesions no larger than $1 \mathrm{~cm}$ in diameter after primary surgery. Importantly, residual disease is the only prognostic factor under the control of the operating surgeon.

Several radical surgical procedures, including intestinal resection, ${ }^{5,6}$ splenectomy, ${ }^{7}$ diaphragmatic resection, ${ }^{8}$ and hepatic resection, ${ }^{9}$ have been described as treatments for advanced ovarian cancer with acceptable morbidity. ${ }^{10}$ Nevertheless, Nguyen et $\mathrm{al}^{11}$ found that only $42-45 \%$ of patients primarily treated by a gynecologic oncologist received optimal cytoreduction, despite a less stringent definition of acceptable residual disease. This rate was not markedly different when compared with the rate of optimal cytoreduction achieved by general obstetricians and gynecologists.

A survey was conducted of the membership of the Society of Gynecologic Oncologists ${ }^{12}$ to gain insight into factors responsible for the infrequency of 
optimal cytoreduction. The sites of disease most frequently noted as precluding optimal cytoreduction were the diaphragm, bowel, and portal triad. Ironically, among respondents who achieved optimal cytoreduction (defined as residual disease $\leq 1 \mathrm{~cm}$ ) in less than $50 \%$ of their ovarian cancer patients, $45.5 \%$ of the surgeons cited lack of evidence for improved survival as a primary rationale against performing aggressive surgical resection.

The philosophy against aggressive surgical resection stems from the largely untested hypothesis that the initial extent of advanced disease (or tumor biology) ultimately dictates treatment outcomes. Therefore, when radical procedures are necessary to achieve optimal cytoreduction, they are unlikely to be helpful, despite minimizing residual disease. Carcinomatosis, large-volume ascites, nodal metastasis, upper abdominal involvement, and high histologic grade are predictors of survival. ${ }^{13-16}$ However, the analysis is confounded because these factors frequently result in suboptimal cytoreduction. Thus, a critical question persists: Can aggressive surgical resection improve survival rates for patients with ovarian tumors? Eisenkop and Spirtos ${ }^{10}$ suggested that aggressive surgical resection does not necessarily portend a worse prognosis for patients, once the outcome is adjusted for residual disease. Furthermore, these single-institution data suggested that extensive surgical efforts do improve overall survival rates. ${ }^{10}$

This study examined the survival rates in a cohort of consecutively treated patients with advanced-stage ovarian cancer. Our surgical group favors aggressive surgical resection for ovarian cancer. Thus, we tested the hypothesis that the extent of surgical resection required for optimal cytoreduction affected ovarian cancer patient survival.

\section{MATERIALS AND METHODS}

Mayo Foundation Institutional Review Board approval was obtained for this study. All patients who received a diagnosis of primary epithelial ovarian cancer between January 1, 1994, and December 31, 1998 , were identified from surgical records. Consecutive patients undergoing primary surgical exploration with a postoperative diagnosis of epithelial ovarian cancer were included.

Preoperative medical evaluations were performed for all patients, and each was classified by American Society of Anesthesiologists (ASA) score. Surgical staging and grading were consistent with the International Federation of Gynecology and Obstetrics (FIGO) standards. ${ }^{17}$ Only patients with FIGO stage IIIC ovarian cancer were included in this study.
Patients who underwent surgical exploration at another institution or who received neoadjuvant chemotherapy before surgery were excluded from the study. All patients were scheduled for treatment with firstline postoperative platinum-based chemotherapy (paclitaxel or cyclophosphamide for 6-8 courses, every 3-4 weeks, according to the specific protocols in effect at the time). Operative reports, hospital and outpatient notes, the Mayo Clinic Cancer Center Registry database, and correspondence from referral institutions were abstracted for relevant clinical data.

Disease status was extracted from surgical exploration notes. Patients were first classified by the extent of peritoneal dissemination. Those with tumor nodules diffusely covering the majority of the bowel serosa surfaces and the parietal peritoneum of the abdomen and pelvis were classified as having carcinomatosis. Patients with disease primarily seeding the mesenteric surfaces or deeper tissues, but not the remainder of the visceral or parietal peritoneum, were classified as having mesentery involvement. Independent of the extent of peritoneal disease, we further categorized patients on the basis of tumor involvement with the diaphragm, presence of cul-de-sac obliteration by the tumor, and the volume of ascites.

For statistical analysis, variables regarding patient characteristics were recorded in the following manner: 1) age, less than or equal to 65 years old or more than 65 years old; 2) ASA score 1 or 2 versus 3 or 4 ; 3 ) histologic grade 1 or 2 versus 3 ; and 4) serous histologic subtype versus all other subtypes. For operative time, we used less than or equal to 150 minutes or greater than 150 minutes, and the interval from surgery to chemotherapy had a 28-day cutoff. Residual disease was noted as follows: 1) no residual disease (no gross tumor at the completion of surgery); 2) residual disease smaller than $1 \mathrm{~cm} ; 3$ ) residual disease of 1-2 cm; and 4) residual disease larger than $2 \mathrm{~cm}$. Statistical analysis was performed with the $\chi^{2}$ analysis and logistic regression analysis. Disease-specific overall survival was measured in all cases. Survival curves were plotted with the Kaplan-Meier method and included the log-rank test. The Cox proportional hazards regression model was used for univariate and multivariable analyses. Significant variables from the univariate analysis were included in the multivariable model. Differences were considered statistically significant at $P<.05$. Statistical software (JMP 5.1; SAS Institute Inc, Cary, NC) was used for the analysis.

\section{RESULTS}

A total of 194 consecutive patients with FIGO stage IIIC ovarian cancer met the inclusion criteria for our 
study (Table 1). Mean and median follow-up was 3.5 and 2.7 years, respectively (range $0.02-10.5$ years). During the follow-up period, the median number of procedures was 2 (range 1-11), and the median number of chemotherapy courses was 3 (range $0-14$ ).

Initial surgery was performed for diagnosis, staging, and surgical cytoreduction. The majority of patients had carcinomatosis, diaphragm involvement, cul-de-sac involvement, and ascites (Table 1). The mean operative time was 210 minutes (range 40-480 minutes). Optimal cytoreduction (defined as residual disease $<1 \mathrm{~cm}$ ) was achieved in $67.5 \%$ of the patients

Table 1. Stage IIIC Ovarian Cancer Patient Characteristics, Tumor Features, and Initial Operative Findings $(n=194)$

\begin{tabular}{|c|c|c|}
\hline \multirow{2}{*}{$\frac{\text { Characteristics }}{\text { Age }(y)}$} & \multicolumn{2}{|c|}{ Patients* } \\
\hline & \multirow{2}{*}{\multicolumn{2}{|c|}{$64.4(24-87)$}} \\
\hline Mean (range) & & \\
\hline Median & \multicolumn{2}{|c|}{64} \\
\hline \multicolumn{3}{|l|}{ ASA score } \\
\hline 1 & 7 & $(3.6)$ \\
\hline 2 & 87 & $(44.8)$ \\
\hline 3 & 88 & $(45.4)$ \\
\hline 4 & 7 & $(3.6)$ \\
\hline Unknown & 5 & $(2.6)$ \\
\hline \multicolumn{3}{|l|}{ Tumor features } \\
\hline \multicolumn{3}{|l|}{ Grade } \\
\hline 1 & 1 & $(0.5)$ \\
\hline 2 & 13 & $(6.7)$ \\
\hline 3 & 180 & (92.8) \\
\hline \multicolumn{3}{|l|}{ Histology } \\
\hline Serous & 126 & $(64.9)$ \\
\hline Mucinous & 4 & $(2.1)^{\prime}$ \\
\hline Endometrioid & 18 & (9.3) \\
\hline Clear cell & 7 & (3.6) \\
\hline Transitional cell & 3 & $(1.5)$ \\
\hline Mixed & 17 & $(8.8)$ \\
\hline Seroanaplastic & 17 & $(8.8)$ \\
\hline Müllerian origin & 2 & $(1.0)$ \\
\hline \multicolumn{3}{|l|}{ Cytology } \\
\hline Positive & 178 & $(91.8)$ \\
\hline Negative & 16 & $(8.2)$ \\
\hline \multicolumn{3}{|l|}{ Residual disease $(\mathrm{cm})$} \\
\hline None detectable & 46 & $(23.7)$ \\
\hline$<1$ & 85 & $(43.8)$ \\
\hline $1-2$ & 22 & (11.3) \\
\hline$>2$ & 41 & $(21.1)$ \\
\hline \multicolumn{3}{|l|}{ Extent of disease } \\
\hline Carcinomatosis & 144 & $(74.2)$ \\
\hline Diaphragm involvement & 137 & $(70.6)$ \\
\hline Mesentery involvement & 138 & $(71.1)$ \\
\hline Cul-de-sac involvement & 163 & $(84.0)$ \\
\hline Omentum involvement & 168 & $(86.6)$ \\
\hline Ascites & 160 & $(82.5)$ \\
\hline Mean (range), mL & 2,076 & $(0-12,000)$ \\
\hline Median, $\mathrm{mL}$ & 1,000 & \\
\hline
\end{tabular}

ASA, American Society of Anesthesiologists.

* Values are expressed as n (\%) unless indicated otherwise.
(131/194; 95\% confidence interval [CI] 60.6-73.7\%), and the 5-year disease-specific overall survival rate was $35 \%$ (68/194; 95\% CI 28.7-42.0\%). The specific primary procedures are summarized in Table 2.

Perioperative mortality rate, defined as the percentage of patients who died within 30 days of surgery, was $1.5 \%(3 / 194 ; 95 \%$ CI $0.5-4.4 \%)$. The ages of these 3 patients were 75,76 , and 83 years, and

Table 2. Surgical Procedures Performed

\begin{tabular}{|c|c|c|}
\hline \multirow{2}{*}{$\begin{array}{l}\text { Anatomic Structures and } \\
\text { Surgical Procedures }\end{array}$} & \multicolumn{2}{|c|}{ Patients $(n=194)$} \\
\hline & $\mathbf{n}$ & $\%$ \\
\hline \multicolumn{3}{|l|}{ Uterus } \\
\hline Hysterectomy & 162 & 83.5 \\
\hline Already done & 25 & 12.9 \\
\hline Not done & 7 & 3.6 \\
\hline \multicolumn{3}{|l|}{ Adnexa } \\
\hline Bilateral & 190 & 97.9 \\
\hline Monolateral & 0 & 0 \\
\hline Biopsy & 3 & 1.5 \\
\hline None & 1 & 0.5 \\
\hline \multicolumn{3}{|l|}{ Omentum } \\
\hline Total & 171 & 88.1 \\
\hline Infracolic & 11 & 5.7 \\
\hline Biopsy & 9 & 6 \\
\hline None & 3 & 1.5 \\
\hline \multicolumn{3}{|l|}{ Diaphragm } \\
\hline Excision nodules & 16 & 8.2 \\
\hline Stripping peritoneum & 6 & 3.1 \\
\hline Segmental resection & 1 & 0.5 \\
\hline Cauterization & 8 & 4.1 \\
\hline None & 163 & 84.0 \\
\hline \multicolumn{3}{|l|}{ Peritoneum } \\
\hline Ablation & 7 & 3.6 \\
\hline General cytoreduction & 53 & 27.3 \\
\hline Cauterization & 6 & 3.1 \\
\hline Excision nodules & 21 & 10.8 \\
\hline None & 107 & 55.2 \\
\hline \multicolumn{3}{|l|}{ Pelvic nodes } \\
\hline Systematic lymphadenectomy & 75 & 38.7 \\
\hline Monolateral lymphadenectomy & 5 & 2.6 \\
\hline Sampling & 15 & 7.7 \\
\hline Not done & 99 & 51.0 \\
\hline \multicolumn{3}{|l|}{ Para-aortic nodes } \\
\hline Systematic lymphadenectomy & 74 & 38.1 \\
\hline Up to IMA & 2 & 1.0 \\
\hline Sampling & 8 & 4.1 \\
\hline Not done & 110 & 56.7 \\
\hline Stripping pelvic peritoneum & 91 & 46.9 \\
\hline Left colectomy & 2 & 1.0 \\
\hline Transverse colectomy & 2 & 1.0 \\
\hline Right colectomy & 3 & 1.5 \\
\hline Rectosigmoidectomy & 47 & 24.2 \\
\hline Small bowel resection(s) & 11 & 5.7 \\
\hline Partial gastrectomy & 2 & 1.0 \\
\hline Splenectomy & 12 & 6.2 \\
\hline
\end{tabular}

IMA, inferior mesenteric artery. 
the ASA score was 3 in 2 patients and 4 in the other. None had undergone any aggressive procedure (see classification below). Nine patients $(4.6 \%)$ did not receive chemotherapy because of rapid disease progression, patient refusal, inadequate performance status, or perioperative death; all 9 died within 3.5 months of their surgery date. Based on the intent to treat, these patients were included in the survival analysis.

Although the extent of disease at presentation may affect patient outcome, the amount of surgical resection and subsequent residual disease also influences survival (Figs. 1A and 1B). We examined the effects of different patient variables on survival using the Cox proportional hazards regression model (Ta-
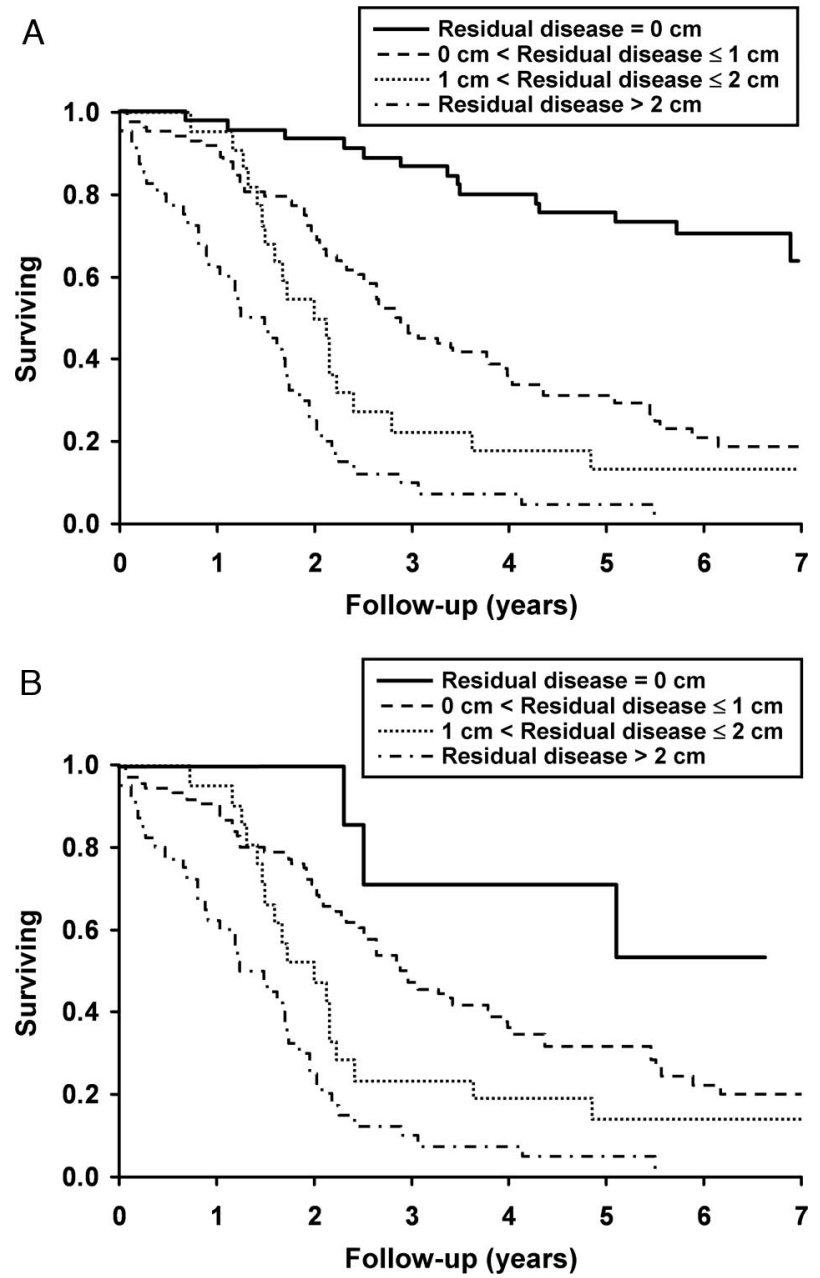

Fig. 1. Residual disease affects survival in patients with stage IIIC ovarian cancer. A. Survival curves for all patients ( $\mathrm{n}=194$; log-rank test, $P<.001$ ). B. Survival curves for patients with carcinomatosis ( $\mathrm{n}=144$; log-rank test, $P<$ .001).

Aletti. Surgery and Advanced Ovarian Cancer. Obstet Gynecol 2005. ble 3). Univariate analysis indicated that age, ASA score, ascites volume, presence of carcinomatosis, disease involvement of the diaphragm or bowel mesentery, and residual disease smaller than $1 \mathrm{~cm}$ were prognostic factors. Multivariate analysis (logistic regression) ultimately showed the only significant predictor of survival was residual disease. We questioned whether the disease-specific overall survival rate for patients with extensive disease was low due to the infrequency of optimal cytoreduction. Not surprisingly, there was a statistically significant direct correlation ( $\chi^{2}$ test) between suboptimal cytoreduction (residual disease $\geq 1 \mathrm{~cm}$ ) and the presence of carcinomatosis $(P<.001)$, diaphragm involvement $(P<$ $.001)$, mesentery involvement $(P<.001)$, or ascites $(P<.001)$. This observation confirmed that the extent of disease affected survival by limiting surgical cytoreduction, but, after adjusting for residual disease, initial tumor volume became a minor determinant of overall survival. Therefore, we hypothesized that patients requiring aggressive surgical procedures to achieve optimal residual disease will have a disease-specific overall survival rate similar to those undergoing relatively less aggressive procedures to achieve the same amount of residual disease. We examined the survival rates of just the 131 patients who had optimal cytoreduction (residual disease $<1 \mathrm{~cm}$ ). If any diaphragmatic surgery, bowel resection, splenectomy, or extensive abdominal peritoneal stripping or resection was performed, the patients were classified in the radical surgery group $(\mathrm{n}=69 ; 53 \%)$. We chose these procedures because they were likely to result in resection of gross disease and are often classified as aggressive treatments. For these reasons, lymphadenectomy was not considered radical surgery. Diaphragm surgery required specific mobilization of liver to achieve exposure for partial-thickness resection of nodules. The remaining patients were classified as having nonradical surgery $(\mathrm{n}=62 ; 47 \%)$. The latter group included patients undergoing hysterectomy, complete omentectomy, stripping of pelvic peritoneum, or limited resection of peritoneal-based nodules. Five-year disease-specific overall survival rates for patients who had aggressive procedures for optimal residual disease were nearly identical to diseasespecific overall survival rates for patients who had optimal residual disease with less aggressive surgery (46\% versus $47 \%$; log-rank test, $P=.80$ ) (Fig. 2). Therefore, optimal cytoreduction, regardless of the extent of surgery, appeared to negate the effect of tumor burden.

To further analyze whether patients undergoing surgical cytoreduction benefit from radical surgery, 
Table 3. Cox Proportional Hazards Regression Model for Different Variables in Patients With Stage IIIC Ovarian Cancer $(n=194)$

\begin{tabular}{|c|c|c|c|c|c|}
\hline \multirow[b]{2}{*}{ Variable } & \multirow[b]{2}{*}{ Risk Ratio } & \multicolumn{2}{|c|}{ Confidence Limit } & \multicolumn{2}{|c|}{$P$} \\
\hline & & Lower & Upper & Univariate & Multivariable* \\
\hline Age $(>$ vs $\leq 65 \mathrm{y})$ & 1.65 & 1.17 & 2.33 & .004 & .12 \\
\hline Age (continuous) & 1.03 & 1.02 & 1.05 & $<.001$ & .56 \\
\hline $\mathrm{ASA}(3$ or 4 vs 1 or 2$)$ & 1.70 & 1.21 & 2.41 & .002 & .79 \\
\hline Ascites $(>$ vs $\leq 1,000 \mathrm{~mL})$ & 2.30 & 1.52 & 3.56 & .001 & .47 \\
\hline Carcinomatosis ( $\mathrm{Y}$ vs $\mathrm{N})$ & 3.63 & 2.32 & 5.97 & $<.001$ & .50 \\
\hline Diaphragm (Y vs $\mathrm{N})$ & 2.43 & 1.63 & 3.74 & $<.001$ & .89 \\
\hline Bowel mesentery ( $\mathrm{Y}$ vs $\mathrm{N}$ ) & 3.07 & 2.02 & 4.82 & $<.001$ & .99 \\
\hline \multicolumn{6}{|l|}{ Residual disease } \\
\hline$<1 \mathrm{~cm}$ vs $0 \mathrm{~cm}$ & 3.89 & 2.27 & 7.11 & $<.001$ & $<.001$ \\
\hline $1-2 \mathrm{~cm}$ vs $0 \mathrm{~cm}$ & 6.25 & 3.16 & 12.61 & - & - \\
\hline$>2 \mathrm{~cm} \mathrm{vs} 0 \mathrm{~cm}$ & 13.00 & 7.14 & 24.87 & - & - \\
\hline Histologic grade (3 vs 1 or 2 ) & 0.97 & 0.54 & 1.97 & .92 & - \\
\hline Histology (others vs serous) & 0.79 & 0.54 & 1.12 & .77 & - \\
\hline Operative time $(>$ vs $\leq 150 \mathrm{~min})$ & 0.68 & 0.48 & 1.00 & .05 & .41 \\
\hline Time to chemotherapy $(<\mathrm{vs} \leq 28 \mathrm{~d})$ & 1.00 & 0.68 & 1.51 & .99 & - \\
\hline
\end{tabular}

ASA, American Society of Anesthesiologists.

* Significant variables for the univariate analysis were included in the multivariable model.

we examined the subgroup of patients with carcinomatosis, ie, the highest volume of disease, who underwent successful optimal cytoreduction $(\mathrm{n}=82)$. The performance of radical surgery was a significant predictor of improved outcome. For patients with radical and nonradical surgery, the 5-year disease-specific overall survival rate was $44 \%$ compared with $17 \%$, respectively (log-rank test, $P<.001$ ). In addition to radical surgery, residual disease, age, ASA score, and operative time correlated with disease-specific overall survival rate (Table 4). However, in multivariable analysis, only residual disease and radical surgery

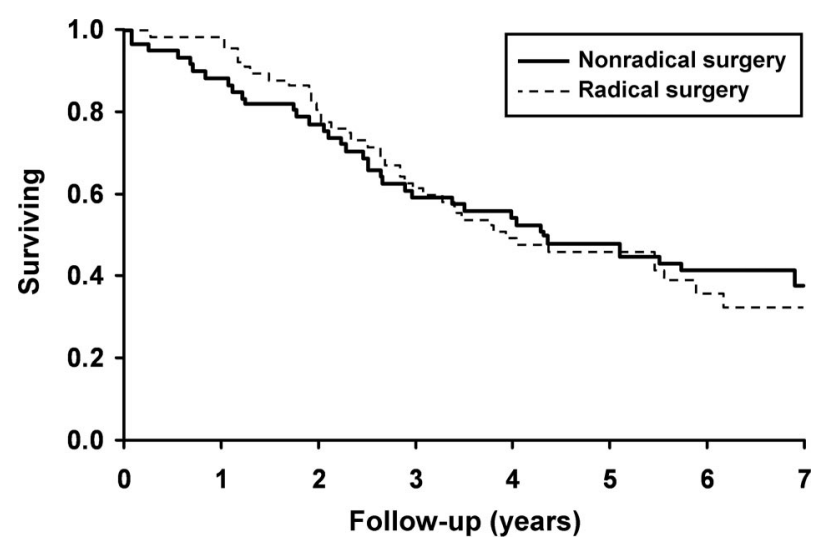

Fig. 2. Effect of surgical effort on Kaplan-Meier survival for patients with stage IIIC ovarian cancer with residual disease smaller than $1 \mathrm{~cm}$. Patients were categorized by surgery required for optimal cytoreduction $(n=131$; log-rank test, $P=.80)$.

Aletti. Surgery and Advanced Ovarian Cancer. Obstet Gynecol 2005. were independent factors predicting patient survival (Table 4).

When examining the effect of radical surgery on all patients with carcinomatosis $(\mathrm{n}=144)$, we observed an improved disease-specific overall survival rate $(38 \%$ versus $9 \%$; log-rank test, $P<.001)$ favoring patients who underwent radical procedures versus nonradical procedures (Fig. 3). This is not unexpected because we anticipated that patients with unresectable disease (or prohibitive medical conditions) would be least likely to undergo radical surgery. Furthermore, we noted a strong correlation between residual disease less than $1 \mathrm{~cm}$ and radical surgery $(P<.001)$. However, as demonstrated earlier, when the analysis was restricted to patients who received optimal cytoreduction, a clear benefit to radical surgery was observed.

We considered factors outside of extent of disease that might be important in the decision to perform radical surgery. Radical procedures were performed at the same rate regardless of age $(49 \%$ for age $\leq 65$ years versus $51 \%$ for age $>65$ years; $P=.45)$. Patients with better ASA scores ( 1 or 2 versus 3 or 4 ) were more likely to have aggressive procedures performed (59\% versus $36 \%$, respectively; $P=.005)$, which implies the overall medical condition of the patient at least partially influences the decision to perform aggressive surgery.

We questioned whether better ASA scores contributed to the survival benefit in patients who underwent radical procedures. We identified a striking correlation among survival rates and the 
Table 4. Cox Proportional Hazards Regression Model for Patients With Stage IIIC Ovarian Cancer and Carcinomatosis at the Beginning of the Surgical Procedure $(n=144)$

\begin{tabular}{|c|c|c|c|c|c|}
\hline \multirow[b]{2}{*}{ Variable } & \multirow[b]{2}{*}{ Risk Ratio } & \multicolumn{2}{|c|}{ Confidence Limit } & \multicolumn{2}{|c|}{$P$} \\
\hline & & Lower & Upper & Univariate & Multivariable* \\
\hline Age $(>$ vs $\leq 65$ y) & 1.15 & 0.80 & 1.67 & .44 & - \\
\hline Age (continuous) & 1.02 & 1.00 & 1.04 & .03 & .73 \\
\hline ASA score (3 or 4 vs 1 or 2$)$ & 1.62 & 1.12 & 2.36 & .01 & .24 \\
\hline $\operatorname{Ascites}(>\mathrm{vs} \leq 1,000 \mathrm{~mL})$ & 1.34 & 0.81 & 2.33 & .26 & - \\
\hline Diaphragm (Y vs N) & 1.11 & 0.66 & 1.98 & .71 & - \\
\hline Bowel mesentery (Y vs $\mathrm{N})$ & 1.64 & 0.88 & 3.48 & .13 & - \\
\hline \multicolumn{6}{|l|}{ Residual disease } \\
\hline$<1 \mathrm{~cm} \mathrm{vs} 0 \mathrm{~cm}$ & 2.55 & 0.94 & 10.47 & $<.001$ & $<.001$ \\
\hline $1-2 \mathrm{~cm}$ vs $0 \mathrm{~cm}$ & 4.05 & 1.36 & 17.28 & - & - \\
\hline$>2 \mathrm{~cm}$ vs $0 \mathrm{~cm}$ & 8.26 & 2.96 & 34.35 & - & - \\
\hline Aggressive surgery (Y vs N) & 0.43 & 0.29 & 0.62 & $<.001$ & .047 \\
\hline Histologic grade ( 3 vs 1 or 2 ) & 1.28 & 0.67 & 2.86 & .49 & .14 \\
\hline Histology (others vs serous) & 0.90 & 0.58 & 1.34 & .60 & - \\
\hline Operative time $(>$ vs $\leq 150 \mathrm{~min})$ & 0.53 & 0.36 & 0.79 & .002 & .34 \\
\hline Time to chemotherapy $(>$ vs $\leq 28 \mathrm{~d})$ & 0.95 & 0.61 & 1.50 & .82 & - \\
\hline
\end{tabular}

ASA, American Society of Anesthesiologists.

* Significant variables from the univariate analysis were included in the multivariable model.

performance of aggressive procedures in the subset of patients with carcinomatosis and good performance status (ASA score $=1$ or $2 ; \mathrm{n}=72$ ). The 5 -year disease-specific overall survival rate was $46 \%$ compared with $13 \%$ for patients with radical and nonradical surgeries, respectively (log-rank test, $P<.001 ;$ Fig. 4A). We restricted the sample population even further by examining only the patients with residual disease smaller than $1 \mathrm{~cm}(\mathrm{n}=49)$. In this analysis (patients with carcinomatosis, ASA score $=1$ or 2 , and residual disease $<1 \mathrm{~cm}$ ), the 5 -year disease-specific overall survival rate was $53 \%$ versus $30 \%$, favoring the aggressive surgery group (log-rank test, $P=.09$; Fig. $4 \mathrm{~B}$ ). Although the

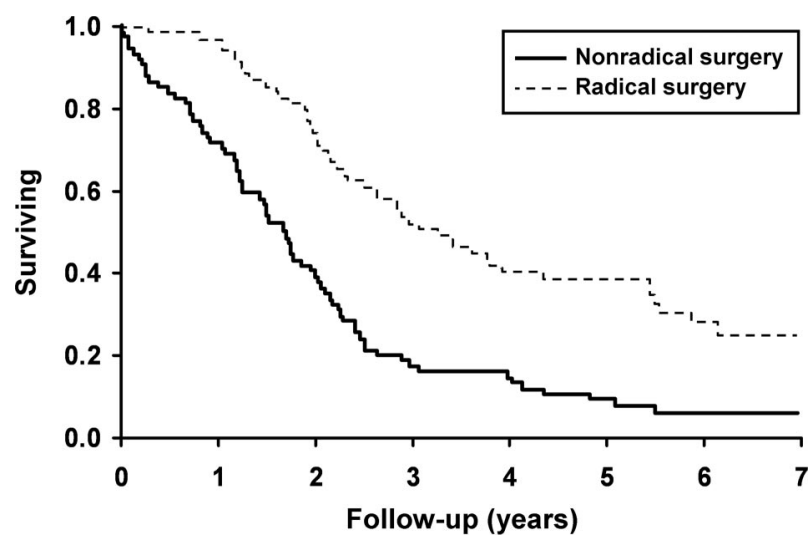

Fig. 3. Effect of surgical resection on Kaplan-Meier survival in patients with stage IIIC ovarian cancer and carcinomatosis ( $\mathrm{n}=144$; log-rank test, $P<.001$ ).

Aletti. Surgery and Advanced Ovarian Cancer. Obstet Gynecol 2005. statistical significance of our observations was limited by the small sample size, these results are suggestive that the benefit of aggressive surgery on survival rate may be independent of performance status.

We wished to estimate whether patient outcomes were affected by a surgeon's propensity to use radical procedures during cytoreductive surgery. Surgeons were classified according to how often they used radical procedures in the management of patients with ovarian cancer. We compared the outcomes for surgeons using radical procedures in more than $70 \%$ of patients and surgeons using radical procedures in less than $40 \%$ of patients (Table 5). There were no significant differences in patient age, ASA score, diaphragm or mesentery involvement, or volume of ascites between groups, confirming a relatively random distribution of patients. However, the rate of optimal resection (residual disease $<1 \mathrm{~cm}$ ) was $84.5 \%$ compared with $51 \%$ on the basis of surgeon tendency to use radical procedures, which clearly highlights the value of extensive surgical effort. Additionally, our division of gynecologic surgery shares a uniform referral base with similar patient demographics, and we practice at a single institution where each surgeon has access to identical services and nursing support. Our analysis indicated that only performance of radical procedures $(81.5 \%$ versus $31.0 \%)$, residual disease, and longer overall operative time substantially affected patient outcomes. We noted striking differences in overall survival for patients treated by surgeons who frequently used radical procedures (Fig. 

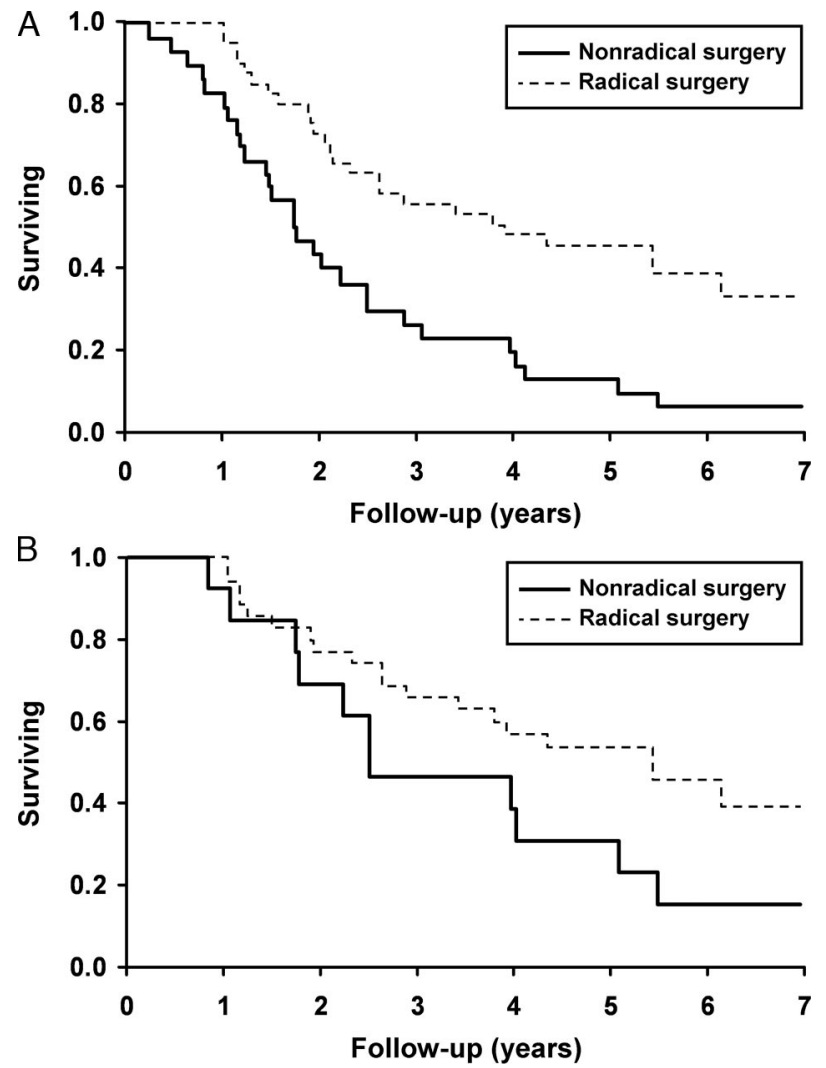

Fig. 4. Effect of surgical resection on Kaplan-Meier survival in patients with stage IIIC ovarian cancer with carcinomatosis and ASA score 1 or 2 . A. All patients $(\mathrm{n}=72$; log-rank test, $P<.001)$. B. Subset of patients with residual disease smaller than $1 \mathrm{~cm}(\mathrm{n}=49$; log-rank test, $P=.09)$.

Aletti. Surgery and Advanced Ovarian Cancer. Obstet Gynecol 2005.

5). These data were the strongest evidence that aggressive surgical resection affected survival for patients with similar disease levels.

\section{DISCUSSION}

Cytoreductive surgery is a cornerstone of the initial treatment for patients with advanced ovarian cancer. The amount of residual disease after primary surgery is generally considered the most important modifiable prognostic factor influencing survival of patients with advanced disease. ${ }^{18}$ In this retrospective study, we observed that 1) residual disease was the only independent predictor of outcome in patients with stage IIIC ovarian cancer; 2) radical surgical resection and residual disease independently affected survival of patients with the most clinically aggressive disease; 3 ) differences among individual surgeons in their tendency to use radical procedures affected overall survival; and 4) considering those patients with optimal cytoreduction (residual disease $<1 \mathrm{~cm}$ ), survival was

the same whether they underwent radical or nonradical surgical procedures.

For stage IIIC ovarian cancer patients, we defined 4 distinct prognostic groups based on residual disease: 1) complete cytoreduction, 2) residual disease smaller than $1 \mathrm{~cm}, 3)$ residual disease between 1 and $2 \mathrm{~cm}$, and 4) residual disease larger than $2 \mathrm{~cm}$. The phrase optimal cytoreduction is clinically vague and disregards the consistent observation that wide differences in survival exist among patients with no residual disease compared with patients having 1 or $2 \mathrm{~cm}$ of residual disease. Although many gynecologic oncologists still define optimal cytoreduction as residual disease smaller than $2 \mathrm{~cm},{ }^{12,19}$ our data support previous evidence that residual disease of $2 \mathrm{~cm}$ has limited validity and that its use in the definition of optimal cytoreduction should be avoided. In addition, care must be taken to prevent confusing the term optimal (intended as a general prognosticator after surgery) with the philosophy that optimal equates with best or is the final goal of cytoreduction. In fact, optimal cytoreduction is an arbitrary designation and not necessarily indicative of the best prognosis. Clearly, the true optimal scenario is complete cytoreduction of visible disease. This should be the goal of cytoreductive surgery for ovarian cancer whenever feasible, mitigated only by the limits of resectability and unacceptable morbidity.

Our results do not deny that tumor biology affects the patient outcome. However, we believe the most valid indicator of tumor aggressiveness is the extent of disease at the initial surgical exploration. Logically, this affects our ability to achieve optimal cytoreduction: our data suggest that tumor aggressiveness has a major effect on survival. The threshold for resectable disease depends on many factors. Nevertheless, even for our patients with the most severe disease, 57\% were resected to less than $1 \mathrm{~cm}$ residual disease. Berman $^{20}$ asserted that it is not understood whether complete cytoreduction reflects the surgeon's skill or the intrinsic biological behavior of the tumor. In fact, the resectability of such disease, with acceptable morbidity, does reflect the surgical experience, technique, excellence in anesthesia, critical care, and nursing. Given the importance of minimizing residual disease, forgoing primary cytoreduction on the basis of the initial tumor extent appears unjustified, at least for practices commonly using radical procedures in the surgical management of ovarian cancer.

Confirming a previous report by Eisenkop and Spirtos, ${ }^{10}$ we observed no difference in disease-specific overall survival rates between patients who required radical surgical procedures to achieve optimal 
Table 5. Comparison of Variables for 144 Patients With Carcinomatosis When Classified by Tendency of Surgeons to Use Aggressive Procedures

\begin{tabular}{|c|c|c|c|}
\hline & \multicolumn{2}{|c|}{ Surgeons (\%) } & \multirow[b]{2}{*}{$P\left(\chi^{2}\right.$ Analysis $)$} \\
\hline & $\begin{array}{c}>70 \% \text { Aggressive } \\
\text { Procedures }(\mathrm{n}=32)\end{array}$ & $\begin{array}{c}<40 \% \text { Aggressive } \\
\text { Procedures }(\mathrm{n}=61)\end{array}$ & \\
\hline Age $(>65 y)$ & 56.0 & 52.5 & .73 \\
\hline \multicolumn{4}{|l|}{ ASA score } \\
\hline 1 & 3.0 & 2.0 & .33 \\
\hline 2 & 59.5 & 40.5 & - \\
\hline 3 & 34.5 & 52.5 & - \\
\hline 4 & 3.0 & 5.0 & - \\
\hline Ascites $(>1,000 \mathrm{~mL})$ & 73.5 & 75.0 & .91 \\
\hline Diaphragm involvement & 84.5 & 82.0 & .87 \\
\hline Mesentery involvement & 97.0 & 85.5 & .06 \\
\hline Aggressive procedures & 81.5 & 31.0 & $<.001$ \\
\hline \multicolumn{4}{|l|}{ Residual disease $(\mathrm{cm})$} \\
\hline 0 & 9.5 & 5.0 & $<.001$ \\
\hline$<1$ & 75.0 & 46.0 & - \\
\hline $1-2$ & 12.5 & 11.5 & - \\
\hline$>2$ & 3.0 & 37.5 & - \\
\hline Operative time $(>150 \mathrm{~min})$ & 94.0 & 63.5 & $<.001$ \\
\hline Histologic grade 3 & 90.5 & 92.0 & .85 \\
\hline Histology, serous & 62.5 & 72.0 & .34 \\
\hline
\end{tabular}

ASA, American Society of Anesthesiologists.

cytoreduction and those who were rendered optimal with nonradical surgery. This further supports the hypothesis that surgical resection minimizes the impact of tumor dissemination. Our data also suggest that radical procedures were superior to nonradical procedures when considering patients with the most extensive disease. The survival benefits did not appear to result from differences in age or ASA status, although we recognize the limitations of our retrospective study in evaluating the complex factors of the

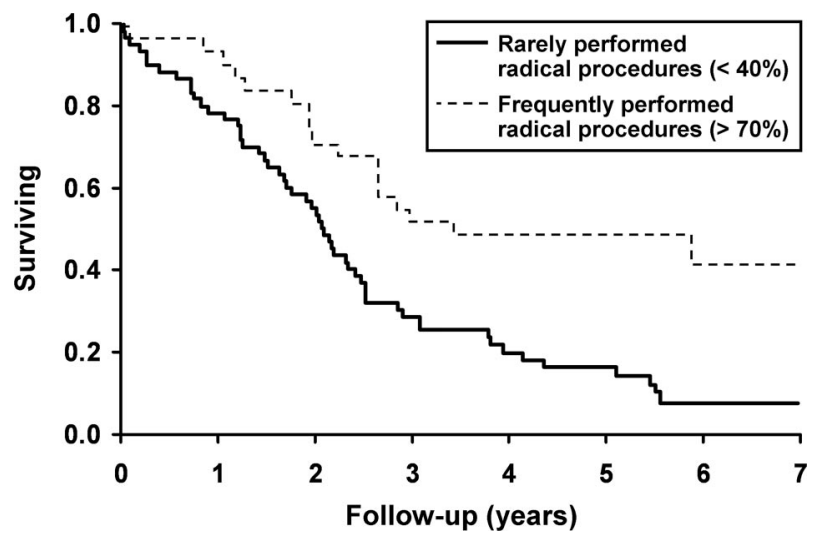

Fig. 5. Effect of surgeon tendency to perform aggressive surgery on Kaplan-Meier survival in patients with stage IIIC ovarian cancer and carcinomatosis (log-rank test, $P<$ .001).

Aletti. Surgery and Advanced Ovarian Cancer. Obstet Gynecol 2005. decision-making process to use radical procedures. Our observation that residual disease and radical surgery independently predicted disease-specific overall survival rates for patients with carcinomatosis strongly suggests that extraordinary surgical efforts positively affect survival for patients with aggressive ovarian cancer. We have several hypotheses that may explain this observation. In the radical surgery subgroup, complete resection of surrounding tissue may remove gross infiltrative or spreading plaques of tumor that could be regarded as optimal if left in situ. Alternatively, the performance of radical procedures was a marker of surgical thoroughness that pervaded throughout the case but was difficult to abstract from operative notes or characterize in general.

Given the consistently observed benefit and the long-held bias in our specialty for the value of cytoreductive surgery, it is unlikely that a randomized trial could be designed to examine the effect of surgical effort on survival. In lieu of this, our retrospective study identified differences in disease-specific overall survival rates that allowed us to examine the influence of surgeons' tendency to use radical procedures on overall survival. Our data originate from a single institution with a common referral pattern, identical intraoperative and postoperative care, and a common practice of adjuvant chemotherapy, all of which add credibility to our findings. Even within a group of surgeons who regularly perform radical procedures to 
achieve low residual disease, we observed large differences in disease-specific overall survival rates based on surgeon tendencies. When extrapolated to practices less likely to use radical procedures, the differences in survival might be much larger.

Because surgical expertise is one of the defining hallmarks of a gynecologic oncologist, it would behoove each center, and perhaps each surgeon, to objectively determine their own rates of surgical cytoreduction for advanced-stage disease. Obstacles to success should be honestly examined with the goal of improving the care of women with ovarian cancer. Given training, concerted efforts, and a coordinated approach to adopting new techniques, substantial improvement in cytoreduction can be achieved, as recently demonstrated by $\mathrm{Chi}$ et $\mathrm{al}^{21}$ at Memorial Sloan-Kettering Cancer Center. Minimal residual disease is a critical factor in determining overall survival and is independent of the radical procedures necessary to achieve this goal, the initial extent of disease, or the biological aggressiveness of the cancer. We should not allow ourselves to dismiss this historically consistent observation without strong evidence that we can predict a priori which tumors will not benefit from resection. This untested hypothesis is unjustified and is unlikely to improve the care of women with ovarian cancer.

\section{REFERENCES}

1. Jemal A, Murray T, Ward E, Samuels A, Tiwari RC, Ghafoor A, et al. Cancer statistics, 2005 [published erratum appears in CA Cancer J Clin 2005;55:259]. CA Cancer J Clin 2005;55: $10-30$.

2. Griffiths CT. Surgical resection of tumor bulk in the primary treatment of ovarian carcinoma. Natl Cancer Inst Monogr $1975 ; 42: 101-4$.

3. Randall TC, Rubin SC. Cytoreductive surgery for ovarian cancer. Surg Clin North Am 2001;81:871-83.

4. Hoskins WJ. Epithelial ovarian carcinoma: principles of primary surgery. Gynecol Oncol 1994;55 suppl:S91-6.

5. Jaeger W, Ackermann S, Kessler H, Katalinic A, Lang N. The effect of bowel resection on survival in advanced epithelial ovarian cancer. Gynecol Oncol 2001;83:286-91.

6. Bristow RE, del Carmen MG, Kaufman HS, Montz FJ. Radical oophorectomy with primary stapled colorectal anastomosis for resection of locally advanced epithelial ovarian cancer. J Am Coll Surg 2003;197:565-74.

7. Chen LM, Leuchter RS, Lagasse LD, Karlan BY. Splenectomy and surgical cytoreduction for ovarian cancer. Gynecol Oncol 2000;77:362-8.

8. Montz FJ, Schlaerth JB, Berek JS. Resection of diaphragmatic peritoneum and muscle: role in cytoreductive surgery for ovarian cancer. Gynecol Oncol 1989;35:338-40.

9. Merideth MA, Cliby WA, Keeney GL, Lesnick TG, Nagorney DM, Podratz KC. Hepatic resection for metachronous metastases from ovarian carcinoma. Gynecol Oncol 2003;89:16-21.

10. Eisenkop SM, Spirtos NM. Procedures required to accomplish complete cytoreduction of ovarian cancer: is there a correlation with "biological aggressiveness" and survival? Gynecol Oncol 2001;82:435-41.

11. Nguyen HN, Averette HE, Hoskins W, Penalver M, Sevin BU, Steren A. National survey of ovarian carcinoma. Part V: the impact of physician's specialty on patients' survival. Cancer 1993;72:3663-70.

12. Eisenkop SM, Spirtos NM. What are the current surgical objectives, strategies, and technical capabilities of gynecologic oncologists treating advanced epithelial ovarian cancer? Gynecol Oncol 2001;82:489-97.

13. Potter ME, Partridge EE, Hatch KD, Soong SJ, Austin JM, Shingleton HM. Primary surgical therapy of ovarian cancer: how much and when. Gynecol Oncol 1991;40:195-200.

14. Farias-Eisner R, Teng F, Oliveira M, Leuchter R, Karlan B, Lagasse $\mathrm{LD}$, et al. The influence of tumor grade, distribution, and extent of carcinomatosis in minimal residual stage III epithelial ovarian cancer after optimal primary cytoreductive surgery. Gynecol Oncol 1994;55:108-10.

15. Silvestrini R, Daidone MG, Veneroni S, Benini E, Scarfone G, Zanaboni F, et al. The clinical predictivity of biomarkers of stage III-IV epithelial ovarian cancer in a prospective randomized treatment protocol. Cancer 1998;82:159-67.

16. Le T, Krepart GV, Lotocki RJ, Heywood MS. Does debulking surgery improve survival in biologically aggressive ovarian carcinoma? Gynecol Oncol 1997;67:208-14.

17. Announcements: FIGO stages-1988 revision. Gynecol Oncol 1989;35:125-7.

18. Ozols RF. Update on the management of ovarian cancer. Cancer J 2002;8 suppl 1:S22-30.

19. Bristow RE, Tomacruz RS, Armstrong DK, Trimble EL, Montz FJ. Survival effect of maximal cytoreductive surgery for advanced ovarian carcinoma during the platinum era: a metaanalysis. J Clin Oncol 2002;20:1248-59.

20. Berman ML. Future directions in the surgical management of ovarian cancer. Gynecol Oncol 2003;90 suppl:S33-9.

21. Chi DS, Franklin CC, Levine DA, Akselrod F, Sabbatini P, Jarnagin WR, et al. Improved optimal cytoreduction rates for stages IIIC and IV epithelial ovarian, fallopian tube, and primary peritoneal cancer: a change in surgical approach. Gynecol Oncol 2004;94:650-4. 